\title{
Cardiac Motion Recovery: Continuous Dynamics, Discrete Measurements, and Optimal Estimation
}

\author{
Shan Tong ${ }^{1}$ and Pengcheng Shi ${ }^{1,2}$ \\ ${ }^{1}$ Department of Electronic and Computer Engineering, \\ Hong Kong University of Science and Technology, Hong Kong \\ ${ }^{2}$ School of Biomedical Engineering, Southern Medical University, China*
}

\begin{abstract}
A sampled-data filtering framework is presented for cardiac motion recovery from periodic medical image sequences. Cardiac dynamics is a continuously evolving physiological process, whereas the imaging data can provide only sampled measurements at discrete time instants. Stochastic multi-frame filtering frameworks are constructed to couple the continuous cardiac dynamics with the discrete measurements, and to deal with the parameter uncertainty of the biomechanical constraining model and the noisy nature of the imaging data in a coordinated fashion. The state estimates are predicted according to the continuous-time state equation between observation time points, and then updated with the new measurements obtained at discrete time instants, yielding physically more meaningful and more accurate estimation results. Both continuousdiscrete Kalman filter and sampled-data $H_{\infty}$ filter are applied, and the $H_{\infty}$ scheme can give robust estimation results when the noise statistics is not available a priori. The sampled-data estimation strategies are validated through synthetic data experiments to illustrate their advantages and on canine MR phase contrast images to show their clinical relevance.
\end{abstract}

\section{Introduction}

Myocardial kinematics reveals critical information about cardiac physiology and pathology, and accordingly there have been abundant efforts devoted to cardiac motion recovery from medical image sequences [1]. Cardiac dynamics is a physical/physiological process which evolves continuously in time, consisting of electrical propagation, electromechanical coupling, and biomechanics-based tissue deformation. However, the imaging/imaging-derived measurements can provide only sampled measurements at discrete time instants, such as computed tomography (CT) images, magnetic resonance (MR) images and ultrasound (US) images. In consequence, the kinematics estimation should be performed on a hybrid system with continuous-time dynamics and discrete-time measurements.

\footnotetext{
* Thanks to IBM PhD fellowship for supporting Shan Tong. This work is supported in part by China National Basic Research Program(973-2003CB716100), National Natural Science Foundation of China(60403040) and by Hong Kong Research Grants Council(CERG-HKUST6151/03E).
} 
Incorporating uncertainties is also essential in cardiac image analysis. As additional constraining models are needed for the ill-posed motion recovery problem, system uncertainties should be incorporated into the constraining models to obtain the flexibility of dealing with subject-dependent data sets. On the other hand, due to the noisy nature of the imaging/imaging-derived measurements, data uncertainties also need to be properly considered.

Formulating the cardiac image analysis problem in a hybrid system, our task is to construct an estimation framework so as to couple the continuous cardiac dynamics with the discrete imaging-derived measurements, and to deal with the system uncertainty of a priori constraining models and the data uncertainty of a posteriori noisy measurements in a coordinated effort. Multi-frame filtering strategies have been applied to tackle this problem. In previous works [2, 3, the system dynamics is first converted to discrete time to perform the analysis, but such conversion involves several assumptions and approximations, which are unrealistic in most real cases, especially for large sampling interval $T$.

In this paper, we propose a sampled-data filtering framework for kinematics estimation of the hybrid cardiac analysis system. Instead of discretizing the system dynamics, state estimates are predicted according to the original continuous state equation and then updated with new measurements at discrete observation time points, which is physically more meaningful for the continuously evolving cardiac dynamics. As approximation errors in the discretization are avoided, estimates of higher accuracy are obtained. Both minimum-mean-squared-error (MMSE) and mini-max $H_{\infty}$ optimization criteria are adopted to couple the continuous dynamics with the discrete observations, and accordingly continuous-discrete Kalman filter and sampled-data $H_{\infty}$ filter are applied for motion recovery. Synthetic experiments have been conducted to validate the advantages of sampled-data filtering and the robustness of $H_{\infty}$ strategy. Results from canine MR images are also presented to show the clinical relevance of this framework.

\section{Methodology}

\subsection{Continuous Biomechanics-Based Myocardial Dynamics}

In biomechanics-based motion analysis, the structure and material of the myocardium should be properly modeled. As it has been shown that the material properties along and cross the myofibers are substantially different [4, we adopt the linear anisotropic material model for a realistic nonrigid motion recovery.

Under spatio-temporal biomechanical constrains, the dynamic equation for myocardium is derived with the principle of minimum potential energy [2]

$$
M \ddot{U}+C \dot{U}+K U=R
$$

where $M, C$ and $K$ are the mass, damping and stiffness matrices respectively, $R$ is the load vector, and $U$ is the displacement vector. $K$ is related to the material-specific Young's modulus, Poisson's ratio, and fiber orientations 4. Rayleigh damping is assumed with $C=\alpha M+\beta K$. 
To apply filtering strategies to our estimation problem, the dynamic equation (11) is transformed into a state-space representation of a continuous-time system:

$$
\dot{x}(t)=A(t) x(t)+B(t) w(t)+\tilde{v}(t)
$$

where the state vector $x(t)$, the input vector $w(t)$, the system matrix $A(t)$, and the input gain $B(t)$ are as follows:

$x(t)=[U(t), \dot{U}(t)]^{\prime}, w(t)=[0, R(t)]^{\prime}, A(t)=\left[\begin{array}{cc}0 & I \\ -M^{-1} K-M^{-1} C\end{array}\right], B(t)=\left[\begin{array}{ll}0 & 0 \\ 0 & M^{-1}\end{array}\right]$,

and $\tilde{v}(t)$ is the process noise describing the disturbances/uncertainties in cardiac dynamics which come from the external load and the subject-dependent material properties. As the material model parameters and the geometry of heart will change over time, this state-space equation represents a time-varying system.

\subsection{Discrete Measurements from Medical Imaging Data}

Although cardiac dynamics has a continuously evolving nature, medical imaging data can provide available measurements $y(k)$ only at discrete observation time instants, which are expressed in a discrete-time measurement equation:

$$
y(k)=D x(k)+e(k)
$$

where $D$ is the measurement matrix, $e(k)$ is the discrete-time measurement noise accounting for the imaging data uncertainties, and $k$ is used to denote the observation time instant $t=k T$. $D$ describes the relationship of the sampled system states $x(k)$ and the discrete imaging data $y(k)$, and many different kinds of data can be incorporated by proper choices of $D$. In our case, the imaging data provides the displacement and velocity information, which can be obtained from $\mathrm{MR}$ tagging data and MR phase contrast images respectively.

\subsection{Optimal Motion Estimation: A Sampled-Data Filtering Framework}

From the above analysis, cardiac image analysis should be performed on a hybrid system with continuous-time dynamics and discrete-time measurements, and our goal is to construct a filtering framework so as to estimate the states of the continuous dynamics given the sampled measurements in some optimal senses.

In order to obtain accurate and physiologically meaningful estimates for the continuous cardiac dynamics, we propose a sampled-data filtering framework for kinematics estimation in such a hybrid system described by equations (2) and (3). In contrast to the system discretization approach in 2, 3, (a detailed comparison is presented in 2.4), the estimate in the sampled-data filtering framework is given directly in terms of the continuous dynamics and the sampled measurements. Without discretizing equation (2), the state estimates are propagated according to the original continuous-time state equation and then updated with new measurements at discrete observation time instants. Both MMSE and mini$\max H_{\infty}$ optimization criteria are adopted, and accordingly continuous-discrete Kalman filter and sampled-data $H_{\infty}$ filter are applied for the motion recovery. 


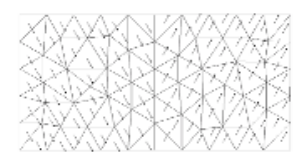

(a)

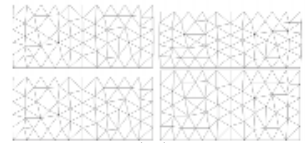

(b)

Fig. 1. (a): Material composition with the arrows denoting fiber orientations. (b): Deformed geometry at selected frames \#4, \#8, \#12, \#16.

Continuous-Discrete Kalman Filtering. Assuming process and measurement noises $\tilde{v}(t)$ and $e(k)$ to be Gaussian distributed, zero-mean and white $\left(E[\tilde{v}(t)]=0, E\left[\tilde{v}(t) \tilde{v}(\tau)^{\prime}\right]=\tilde{Q}(t) \delta(t-\tau), E[e(k)]=0, E\left[e(k) e(j)^{\prime}\right]=V(k) \delta_{k j}\right)$, continuous-discrete Kalman filter yields the optimal estimate in the MMSE sense.

Continuous-discrete Kalman filter is a recursive filter consisting of a prediction stage and an update stage [5. In the prediction stage, i.e. between two observation time instants, the state estimate $\hat{x}(t)$ and its covariance $P(t)$ propagate according to the following two prediction equations [5]:

$$
\begin{aligned}
\dot{\hat{x}}(t) & =A(t) \hat{x}(t)+B(t) w(t) \\
\dot{P}(t) & =A(t) P(t)+P(t) A(t)^{\prime}+\tilde{Q}(t)
\end{aligned}
$$

Define $\hat{x}\left(k T^{-}\right)=\lim _{\varepsilon \rightarrow 0} \hat{x}(k T-\varepsilon)$. The predicted state $\hat{x}\left(k T^{-}\right)$and its associated covariance $P\left(k T^{-}\right)$can be obtained by solving equations (4) and (5) on the time interval $[(k-1) T, k T]$, with the previous state estimate $\hat{x}((k-1) T)$ and covariance $P((k-1) T)$ as the initial conditions of the differential equations.

In the update stage, i.e. at the observation time $t=k T$ with new measurements available, the state estimate and its covariance are updated by the standard discrete Kalman filter as follows:

$$
\begin{aligned}
\hat{x}(k T) & =\hat{x}\left(k T^{-}\right)+W(k)\left[y(k)-D \hat{x}\left(k T^{-}\right)\right] \\
P(k T) & =P\left(k T^{-}\right)-W(k) S(k) W(k)^{\prime}
\end{aligned}
$$

where $S(k)=D P\left(k T^{-}\right) D^{\prime}+V(k)$, and the Kalman gain $W(k)=P\left(k T^{-}\right) D^{\prime} S(k)^{-1}$.

Sampled-Data $\boldsymbol{H}_{\infty}$ Filtering. In practical situations, the noise statistics is usually not available a priori and the Gaussian assumption in Kalman filter may not be valid. To relax such restrictions, the mini-max $H_{\infty}$ strategy is adopted in the sampled-data filtering framework to achieve robustness in motion recovery.

The performance measure of sampled-data $H_{\infty}$ filter is defined directly in terms of the continuous-time system state $x(t)$ and disturbance $\tilde{v}(t)$ :

$$
J=\frac{\|x(t)-\hat{x}(t)\|^{2}}{\|\tilde{v}(t)\|^{2}+\|e(k)\|^{2}+\left(x_{o}-\hat{x}_{o}\right)^{\prime} R_{0}\left(x_{0}-\hat{x}_{o}\right)}
$$

and thus inter-sample behavior of the system is taken into account. The weighting matrix $R_{0}$ is a confidence measure of the initial state estimate $\hat{x}_{o}$. 
Table 1. Differences between the ground truth and the DKF $/$ CDKF $/ H_{\infty}$ estimated nodal positions, (mean error \pm standard deviation) $\times 10^{-2}$

\begin{tabular}{|c|c|c|c|c|}
\hline Method & 20dB(Gaussian) & 30dB(Gaussian) & 20dB(Poisson) & 30dB(Poisson) \\
\hline DKF & $1.39 \pm 0.47$ & $1.21 \pm 0.37$ & $2.73 \pm 0.59$ & $2.41 \pm 0.53$ \\
\hline CDKF & $1.27 \pm 0.43$ & $1.09 \pm 0.34$ & $2.56 \pm 0.54$ & $2.27 \pm 0.51$ \\
\hline$H_{\infty}$ & $1.29 \pm 0.44$ & $1.18 \pm 0.37$ & $1.30 \pm 0.42$ & $1.21 \pm 0.37$ \\
\hline
\end{tabular}

Table 2. Comparison of average nodal positional errors from sampled-data $H_{\infty}$ filter results under various types of noise, (mean error \pm standard deviation) $\times 10^{-2}$

\begin{tabular}{|c|c|c|c|c|c|}
\hline Noise Type (20dB) & Gaussian & Poisson & Uniform & Rayleigh & Exponential \\
\hline Average Positional Error & $1.29 \pm 0.44$ & $1.30 \pm 0.42$ & $1.32 \pm 0.43$ & $1.33 \pm 0.45$ & $1.29 \pm 0.41$ \\
\hline
\end{tabular}

Given a prescribed noise attenuation level $\gamma>0$, the sampled-data $H_{\infty}$ filter will search for the optimal estimate $\hat{x}(t)$ such that

$$
\sup J \leq \gamma^{2}
$$

where the supremum is taken over all possible disturbances and initial states. The formulation in equation (9) guarantees the bounded estimation error over all possible disturbances, regardless of the noise statistics. As a result, the filter achieves greater robustness to disturbance variations and is well suited to such real-world problems as in cardiac image analysis, where the types and levels of system disturbances and data uncertainties are not available a priori.

The sampled-data $H_{\infty}$ filtering algorithm for the hybrid cardiac analysis system of equations (2) and (3) is given as follows [6]:

$$
\begin{aligned}
\dot{\hat{x}}(t) & =A(t) \hat{x}(t)+B(t) w(t) \\
\hat{x}(k T) & =\hat{x}\left(k T^{-}\right)+P(k T) D^{\prime}\left[y(k)-D \hat{x}\left(k T^{-}\right)\right]
\end{aligned}
$$

where $\hat{x}\left(k T^{-}\right)=\lim _{\varepsilon \rightarrow 0} \hat{x}(k T-\varepsilon)$, and $P(k T)$ is the stabilizing solution to the following Riccati equation with jumps:

$$
\begin{aligned}
\dot{P}(t) & =A(t) P(t)+P(t) A(t)^{\prime}+\frac{P(t)^{2}}{\gamma^{2}}+I \\
P(k T) & =P\left(k T^{-}\right)\left[I+D^{\prime} D P\left(k T^{-}\right)\right]^{-1}
\end{aligned}
$$

with the initial condition $P\left(0^{-}\right)=R_{0}^{-1}$.

The filter given above is a linear system with finite jumps, which also has an intuitively appealing structure. Between the sampling instants, the state estimate evolves according to the continuous system dynamics, and the predicted state $\hat{x}\left(k T^{-}\right)$is obtained by solving the differential equation (10) on the time interval $[(k-1) T, k T]$, with the previous state estimate $\hat{x}((k-1) T)$ as initial condition. Then at the observation time $t=k T$, the new measurement $y(k)$ is used to update the estimate with the filter gain being $P(k T) D^{\prime} . P\left(k T^{-}\right)$is also obtained by solving the differential equation (12) with $P((k-1) T)$ as initial condition. 


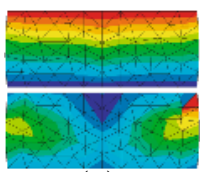

(a)

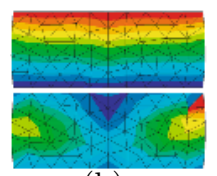

(b)

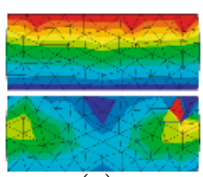

(c)

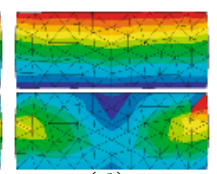

(d)

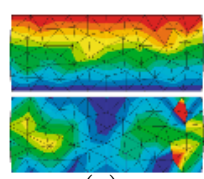

(e)

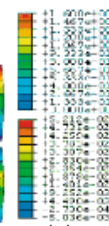

(f)

Fig. 2. Results comparison at frame \#8 (the largest deformation time). Top: displacement magnitude maps. Bottom: x-strain maps. (a): ground truth. (b)(c): estimation results under Gaussian noise. (b): CDKF results, (c): DKF results. (d)(e): estimation results under Poisson noise. (d): $H_{\infty}$ results, (e): CDKF results. (f): color scale.

Computational Issues. Numerical integration is usually required for solving Riccati differential equation (12), and its property of movable singularities usually leads to stability problems when Runge-Kutta routines are applied. The Möbius schemes proposed in [7] are based on viewing the Riccati equation as a flow on the Grassmannian. Since there are no singularities in the associated flow, the schemes are able to deal with numerical instability and pass accurately through the singularities. Thus we adopt Möbius schemes in our implementation, and the detailed algorithm can be found in [7]. The Lyapunov differential equation (5) is a special case of equation (12), and is solved in the same way.

\subsection{Discussions}

In [2, 3], the continuous-time state equation (2) in the hybrid system is first converted to discrete time and discrete Kalman or $H_{\infty}$ filters are applied for kinematics estimation. After discretization, the state equation becomes:

$$
x(k+1)=F(k+1, k) x(k)+G(k) w(k)+v(k)
$$

where the transition matrix $F(k+1, k)=e^{A T}, G(k)=A^{-1}\left(e^{A T}-I\right) B$, and $v(k)$ is the discrete process noise.

There are several implicit approximations/assumptions in the above conversion. First, the assumption that the input is piecewise constant is required. Second, the transition matrix $F(k+1, k)$ in general has no explicit form, and it can be reduced to the matrix exponential form $e^{A T}$ only when the system is time-invariant [5]. In consequence, the accuracy of the estimate from discrete Kalman and $H_{\infty}$ filters largely depends on the extent to which the discretized state equation (14) approximates the true continuous dynamic equation (2). For large sampling interval $T$, the assumptions of piecewise constant input and time-invariant system become unrealistic, and thus equation (14) is not a faithful representation of the original continuous-time system dynamics.

In contrast to the system discretization approach, sampled-data filtering evolves the state estimate and the filter gain according to the original continuous system dynamics through equations (4) (5) in Kalman filter and equations (10) (12) in $H_{\infty}$ filter, and thus physically more meaningful estimates could be 


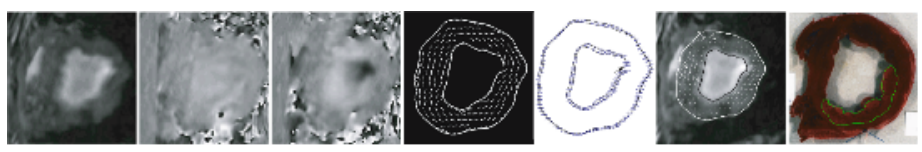

Fig. 3. Canine MRI phase contrast data (left to right): MR intensity, x-velocity, yvelocity, myofiber orientations, displacement constraints on the boundaries, phase contrast velocity field, and TTC-stained post mortem myocardium with infarcted tissue highlighted (providing the clinical gold standard for assessing image analysis results).

obtained for the continuous cardiac dynamics. And as the approximation errors in the discretization are avoided, the estimation results would be more accurate.

Sampled-data Kalman and $H_{\infty}$ filters are closely related to each other. The state prediction equations (4) and (10) in the two filters are essentially the same, which is consistent with the idea that the state estimates should evolve according to the original continuous dynamics between observations, regardless of the optimization criterion. Moreover, as $\gamma \rightarrow \infty$, the Riccati equation (12) becomes the Lyapunov equation (5) despite the different weighting matrix $\tilde{Q}(t)$, and thus Kalman filter can be regarded as a limiting case of $H_{\infty}$ filter when $\gamma \rightarrow$ $\infty$. As a result, the estimation error under the worst disturbances is unbounded in Kalman filter, making it sensitive to parameter variations and noise statistics violations. In contrast, $H_{\infty}$ estimation minimizes the maximum error energy over all possible disturbances, thus demonstrating greater robustness.

\section{Experiments and Validation}

\subsection{Validation with Synthetic Data}

Experiments are conducted on an object undergoing deformation in the vertical direction with the bottom being fixed. In Fig 1(a), the object is composed of two materials with different fiber orientations, and the Young's modulus $E_{\text {fiber }}=75$, $E_{\text {crossfiber }}=25$. Sixteen frames of the cyclic motion are acquired (Fig 1 (b)), and the boundary nodes displacements are added with different types and levels of noise to generate the partial and noisy measurements. Three estimation strategies: continuous-discrete Kalman filter (CDKF), discrete Kalman filter (DKF), and sampled-data $H_{\infty}$ filter $\left(H_{\infty}\right)$, are implemented for motion recovery. Fig 2 shows the estimated displacement magnitude and strain maps at frame \#8.

The advantages of sampled-data filtering are validated through the comparison between CDKF and DKF in Fig_2(b)(c) and Table1. CDKF results are overall closer to ground truth and smoother than DKF results, validating our analysis in section 2.4. Without system discretization, CDKF avoids the approximation errors and yields more accurate estimates under the same noise level.

The robustness of $H_{\infty}$ strategy is validated through the results in Fig 2(d)(e), where the noise is Poisson distributed. For quantitative comparison, Table 1 and 2 present the point-by-point positional errors under noises of different types and levels. Overall, CDKF performs poorly if the Gaussian noise assumptions are 


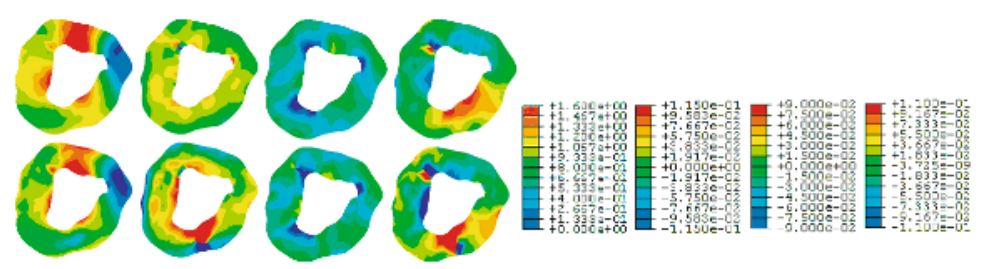

Fig. 4. Estimated displacement magnitude, radial (R), circumferential (C), and RC shear strain maps for frame \#9 (with respect to frame \#1). Top: CDKF results. Bottom: $H_{\infty}$ results. Right: color scales.

violated, while $H_{\infty}$ filter produces accurate and very similar results for data contaminated by different types and levels of noise.

\subsection{Canine Image Application}

For the MR phase contrast data in Fig 3 . myocardial boundaries and frame-toframe boundary displacements are extracted using a unified active region model strategy [8], and the displacements are used as our measurements along with the mid-wall phase contrast velocities. The myofiber orientations are mapped onto the particular image slice, based on the principal warps algorithm of the landmarks. Fig 4 presents the recovered displacement and strain maps from sampleddata $H_{\infty}$ filter and CDKF. The lower-right quarter of the myocardium shows much larger strain than other normal tissues, especially in the RC strain maps. These patterns are in good agreement with the highlighted TTC stained tissue in Fig 3. The computational cost is hindering the experiments for 3D cases, and we are looking into possibilities such as modal decomposition to tackle the problem.

\section{References}

1. Frangi, A., Niessen, W., Viergever, M.: Three-dimensional modeling for functional analysis of cardiac images: A review. IEEE TMI 20(1) (2001) 2-25

2. Shi, P., Liu, H.: Stochastic finite element framework for simultaneous estimation of cardiac kinematic functions and material parameters. Medical Image Analysis 7 (2003) 445-464

3. Liu, H., Shi, P.: Cardiac motion and elasticity characterization with iterative sequential $\mathrm{H} \infty$ criteria. In: MICCAI. (2004) 34-42

4. Glass, L., Hunter, P., McCulloch, A.: Theory of heart. Springer (1991)

5. Bar-Sharlom, Y., Li, X., Kirubarajan, T.: Estimation with applications to tracking and navigation. Wiley (2001)

6. Sun, W., Nagpal, K.M., Khargonekar, P.: H $\infty$ control and filtering for sampled-data systems. IEEE TAC 38 (1993) 1162-1175

7. Schiff, J., Shnider, S.: A natural approach to the numerical integration of riccati differential equations. SIAM Journal on Numerical Analysis 36 (1996) 1392-1413

8. Wong, L., Liu, H., Sinusas, A., Shi, P.: Spatio-temporal active region model for simultaneous segmentation and motion estimation of the whole heart. In: IEEE VLSM. (2003) 193-200 